\title{
Correction to: Why was Schumpeter not more concerned with patents?
}

\section{Rémy Guichardaz ${ }^{1} \cdot$ Julien Pénin ${ }^{1}$}

Published online: 29 August 2019

C) Springer-Verlag GmbH Germany, part of Springer Nature 2019

\section{Correction to: Journal of Evolutionary Economics https://doi.org/10.1007/s00191-019-00633-y}

The original version of this paper unfortunately contained error. The names of the authors are inadvertently inverted. The correct names are already corrected in this paper.

\author{
$<$ GivenName $>$ Rémy $<$ /GivenName $>$ \\ $<$ FamilyName $>$ Guichardaz $<$ FamilyName $>$ \\ $<$ GivenName $>$ Julien $<$ GivenName $>$ \\ $<$ FamilyName $>$ Pénin $<$ /FamilyName $>$
}

The original version of this article is corrected.

Publisher's note Springer Nature remains neutral with regard to jurisdictional claims in published maps and institutional affiliations.

The online version of the original article can be found at https://doi.org/10.1007/s00191-019-00633-y

Rémy Guichardaz

rguichardaz@unistra.fr

Julien Pénin

penin@unistra.fr

1 Université de Strasbourg, Université de Lorraine, CNRS, BETA, 67000 Strasbourg, France 